\title{
Update on early thrombus removal for iliofemoral deep vein thrombosis
}

\author{
Update: Frühe Thrombus-Entfernung aus den Ilio-Femoralvenen
}

Author

Niels Bækgaard

Affiliations

Associate professor emeritus, Vascular Clinic, Gentofte Hospital and Rigshospitalet, University of Copenhagen, Denmark

Key words

Iliofemoral deep vein thrombosis, endovenous thrombus removal, surgical thrombectomy, post-thrombotic syndrome

\section{Schlüsselwörter}

Iliofemoralvenenthrombose, endovenöse Thrombectomie, chirurgische Thrombusentfernung, postthrombotisches Syndrome

received 17.03 .2019

accepted 20.03.2019

Bibliography

DOI https://doi.org/10.1055/a-0893-4654

Published online: 22.05.2019

Phlebologie 2019; 48: 228-236

(c) Georg Thieme Verlag KG Stuttgart · New York ISSN 0939-978X

Correspondence

MD Niels Bækgaard

Associate professor emeritus Niels Bækgaard

Vascular Clinic, Gentofte Hospital and Rigshospitalet, University of Copenhagen, Denmark

E-Mail: baekgaard@dadlnet.dk

\section{ABSTRACT}

Thrombus removal has for many years been attractive to investigate. The reasons were to relieve the acute symptoms with painful swelling and even to avoid tissue loss, but also with focus to decrease the incidence of late complications. Surgical procedures in the beginning and systemic thrombolysis years later were practiced in few dedicated centers. The breakthrough came 30 years ago in order to concentrate the lysis procedure directly into the thrombus material. This minimal invasive catheter-directed procedure was soon combined with stenting of the well-known iliac vein compression syndrome. Further development of this modality has taken place with some adjunctive mechanical devices primarily to minimize bleeding, which is the major concern of the methods. Less than ten RCT's have been published over time, but conclusively supporting the rationale of early thrombus removal for iliofemoral DVT with reduction of PTS.

\section{ZUSAMMENFASSUNG}

Die Entfernung der Thromben aus einer Beinvene war über viele Jahre kontrovers diskutiert. Die Ergebnisse wurden untersucht, zum einen mit Blick auf die Entlastung der akuten Symptome einer Thrombose mit Schwellung, Schmerz, bis hin zum Gewebeverlust, aber auch mit dem Ziel der Verringerung später Komplikationen. In der Anfangszeit verfügte man nur über chirurgische Maßnahmen, später folgte die systemische Thrombolyse, diese Maßnahmen waren invasiv und wurden in wenigen spezialisierten Zentren vorgenommen.

Der Durchbruch geschah vor 30 Jahren, als die lokale Thrombolyse mit Wirkung direkt am Thrombose-Material eingeführt wurde. Es handelte sich um einen minimal-invasiven, Kathetergestützten Eingriff, der bald kombiniert wurde mit dem Stenting bei bekannten Iliakal-Venen-Kompressionssyndromen. Es folgten technische Verbesserungen, insbesondere mit mechanischen Techniken, die auf eine Minimierung der bei diesen Maßnahmen immer befürchteten Blutungskomplikationen zielten. Weniger als 10 randomisierte kontrollierte Studien wurden über die Jahre veröffentlicht. Sie stimmen jedoch im Ergebnis überein: Bei einer frühen Entfernung der Thrombose im iliakalen Bereich entwickelt sich weniger häufig ein postthrombotisches Syndrom.

\section{Introduction}

Iliofemoral deep vein thrombosis (DVT) has for many years invoked great attention for many reasons. Even not being the most frequent localization, the disease possesses risk for fatal pulmonary embolism (PE) and serious complications in the post-thrombotic syndrome (PTS). To avoid the latter there has been a persistent in- terest for methods to remove the thrombus material before damage destroys the vein wall irreversibly. The spectrum of these attempts has ranged from surgery, systemic and regional chemical thrombolysis in the past to endovenous catheter-based procedures in the last 2-3 decades. 
- Table 1 Some useful definitions (1).

\section{Clinical presentations, terms and procedures with corresponding definitions}

Iliofemoral deep vein thrombosis

Femoropopliteal deep vein thrombosis

Proximal deep vein thrombosis

Acute DVT

Subacute DVT

Post-thrombotic changes

Post-thrombotic syndrome

Catheter-directed thrombolysis (CDT)

Pharmacomechanical thrombectomy (PMT or PCDT)

Mechanical thrombectomy

Aspiration

Ballooning

Stenting

Surgical thrombectomy
Complete or partial thrombosis of any part of the lliac vein and/or the common femoral vein with or without other associated veins

Complete or partial thrombosis of the popliteal vein, femoral vein and/or deep femoral vein Iliofemoral DVT and/or femoropopliteal DVT

Venous thrombosis for which symptoms have been present for less than 15 days or for which imaging studies indicate that thrombosis occurred within the previous 14 days

Venous thrombosis for which symptoms have been present for 15-28 days indicated by history or imaging studies

Venous thrombosis for which symptoms have been present for more than 28 days as indicated by history or imaging findings

Complication after ipsilateral DVT due to venous symptomatic hypertension secondary to obstruction and/or valvular incompetence earliest diagnosed 3-6 months after the DVT episode

Infusion of a lytic drug through a multi-side-hole catheter with top occlusion given either continuously or with pulsatile injections (pulse-spray). The infusion can be ultrasound enhanced with intension to enlarge the thrombus surface making it more susceptible for lysis

Combines the lytic infusion with a mechanical device, usually with a rheolytic thrombectomy system, which extracts the thrombus via suction

Different devices with ability to remove thrombus

by rotational suction principles, which can be combined with CDT

Refers to syringe aspiration technique (manual), which can be combined with CDT

Dilatation and maceration of (residual) thrombus with a balloon to enlarge the thrombus surface making it more susceptible for lysis

Insertion of a stent (one or more) for treatment of persistent of iliac obstruction with pre- and postballoon dilatation

Refers to an open surgical procedure usually in the groin including thrombus extraction, stenting and sometimes creating an a-v fistula
This article will summarize on the thrombus characteristics, clinical manifestation and provide a review of the procedures for modern thrombus removal with plasminogen activating methods sometimes with mechanical adjunctive devices and modern surgical thrombectomy. Some historical development, but more important, the newest literature with big series and randomized controlled trials (RCT's) will be reviewed in a chronologic order.

\section{Definitions and distribution of iliofemoral DVT}

According to mostly used definition the term iliofemoral DVT is synonymous with DVT in iliac and/or common femoral vein with or without DVT in additional veins, \ Table 1 (1). The hemodynamic consequence is blockage of the venous return from the femoral and/or the deep femoral vein resulting in severe acute signs and later developing of PTS in around $40-70 \%$ of patients over time with worsening of quality of life $(\mathrm{QOL})$ with venous claudication as a dominant finding, usually not identified in the Villalta score $(2-5)$. The reason is less rate of recanalization of the iliac diseased vein due to the iliac vein compression syndrome typically on the left side, $>$ Fig. 2. A more or less permanent outflow obstruction is the result, because as many as $80 \%$ will remain occluded thus 4 -fold more frequent compared to the femoral vein with corre-

\section{Chemical thrombus breakdown}

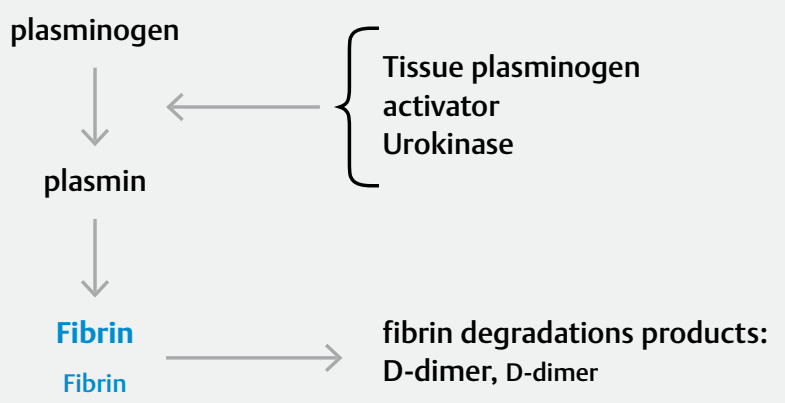

- Fig. 1 Illustration of the chemical breakdown of thrombus. Concentration of D-dimer decreases at the end of sufficient treatment.

sponding lower rate of PTS $(6,7)$. The pathophysiologic consequence of obstruction and/or reflux is venous hypertension, which can be measured directly as the ambulatory venous pressure or indirectly with a non-invasive method using plethysmography, both classical investigations in the venous field, most often used for research only.

A recent report on 1.338 patients, aged $>18$ years, retrospectively analyzed from a single center cohort between 1994-2012 

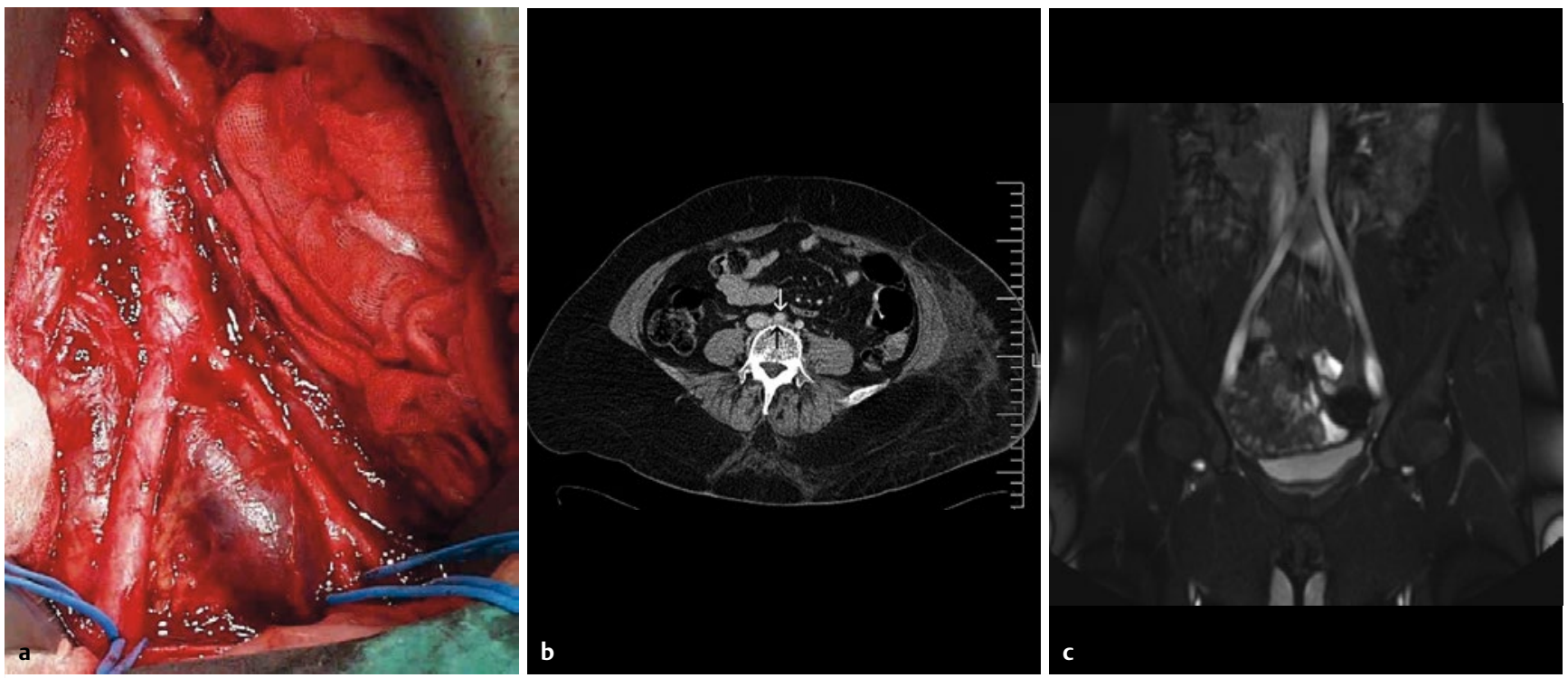

- Fig. 2 The images illustrate the typical left-sided iliac vein compression syndrome. a From the outside with a pre-stenotic dilatation of the left common iliac vein in front of the right common iliac artery. $\mathbf{b}$ The left common iliac vein compressed under the right common iliac artery (the white arrow). $\mathbf{c}$ The left common iliac vein compressed and flattened by the crossing right common iliac artery.

having acute unilateral first-time DVT diagnosed with duplex ultrasound scanning (DUS), showed almost $40 \%$ with involvement of IVC- ilio- common femoral outflow tract. Only $1 / 3$ of these patients had free popliteal vein and thereby optimal for endovenous thrombus removal according to the authors (8).

\section{Thrombus age and composition, clinical manifestation and imaging}

\section{Material, age and composition}

The age of thrombus is crucial to indicate a removal. The older the more difficult to remove and then the vein wall can be damaged. The initial clot of a thrombus is a mixture of red blood cells, fibrin and platelet aggregation producing $\mathrm{P}$ - selectins as the most important adhesion molecules accompanied of perivenous inflammation, pointing out that processes take place in the clot and the vein wall as well. At the same time intrinsic fibrinolysis with uPA and tPA is acting. The inflammatory process continues with influx of polymorph nuclear neutrophils, monocytes and collagen deposition in an interaction with thrombus resolution and at the same time breakdown of elastin and collagen in the vein wall making it inelastic. Furthermore, there is a recruitment of non-contractile muscle cells into a neo-intima and media layer. These very complex actions seem to occur within the time span of very few days and weeks with continuing remodeling changes (9). A detailed composition of the fibrin structure itself has also been investigated, and it seems that alterations in permeability in term of fibrin compactness might hinder thrombolysis, an aspect needed to be explored more in the future in correlation to outcome after catheter-directed thrombolysis (CDT) (10). Further discussion concerning ongoing post-thrombotic cellular processes lies without the scope of this chapter.

\section{Clinical manifestation}

There are typical signs for an iliofemoral DVT. The pain often begins in the back and then moves distally when the extremity becomes swollen from the groin whereas the other way around, with crural pain extending in a proximal direction, is less frequent. The condition is called phlegmasia alba dolens. The leg is pale with milky appearance and painful. The pain is due to both the venous dilatation and inflammatory response. The acute patient is often hampered in walking with contraction around the hip and needs to be bedridden. The extremity is not circulatory threatened, because there is some venous return mainly from the superficial and collateral systems. The clinical outcome is much worse if the entire axial, superficial and collateral veins are thrombosed now identical with phlegmasia cerulea dolens. The extremity is extreme painful, bluish with congestion and often includes crural compartment syndrome, which can develop into total micro-arterial collapse with peripheral gangrene and tissue loss. This type of patient is in a very time-sensitive situation, because the situation can develop over few hours. The condition can be seen even in otherwise healthy persons, but more often in patients with cancer or with severe hypercoagulable diseases. This condition is not necessarily preceded by the alba appearance.

In the prospective TULIPA registry with 135 patients having DVT a crural swelling $>3 \mathrm{~cm}$ larger than the asymptomatic leg (HR 2.94; $95 \% \mathrm{Cl} 1.20-7.20$ ) remained predictive for PTS at 3 years follow-up, showing how important this sign is and why it is obligatory in any PTS score (11).

\section{Imaging}

The golden diagnostic standard is duplex ultrasound scanning (DUS) with B-mode imaging and Doppler aided or not with color flow assessment typical in cross-sectional images with the patient in different lying positions to ensure the findings. The compression maneuver is the primary test to be done. A fully occluding fresh 
- Table 2 Contraindications for endovenous thrombus removal with CDT/PMT/PCDT (1).

Absolute contraindications

Active internal bleeding or disseminated intravascular coagulation

Recent cerebrovascular events or intracranial trauma ( $<3$ months)

Absolute contraindication to anticoagulation

Non-cooperative patients
Relative contraindications

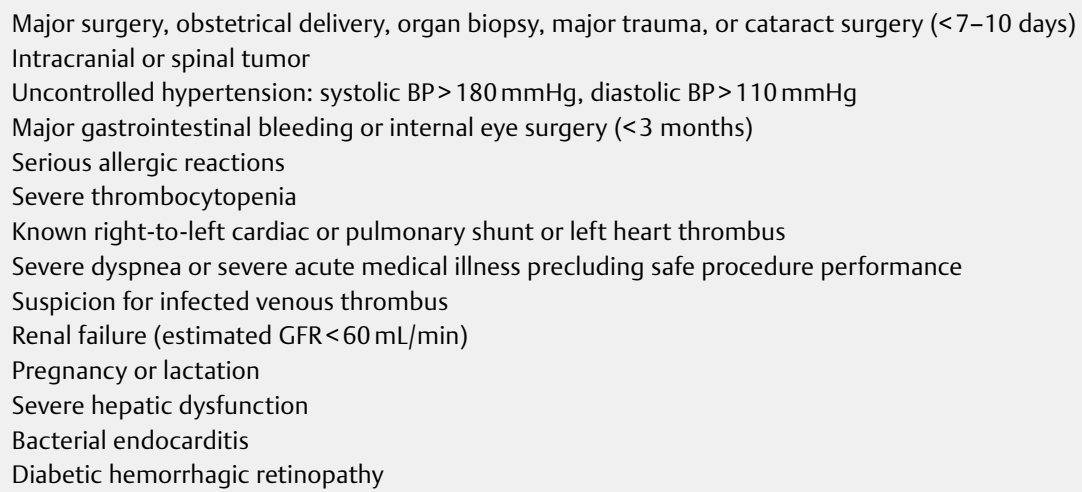

thrombus enlarges the vein and hinder the vein to collapse under probe-compression. A partly thrombosed vein acts different: the vein can be compressed in some degree around the thrombus, but the thrombus is still impossible to compress. The acute clot structure is echolucent (black), but changes after few days into more and more echogenic grey color due to the fibro-cellular resolution and reorganization. Some data have looked at the movement in the interface between the clot and vein wall. After in average 11-12 days this interface does seem "fixed", meaning a stage with irreversible vein wall damage might occur (6). Subsequently, the thrombus shrinks, fragmentizes and recanalizes. A routine DUS is often only looking at the popliteal and the groin region. However, with more and more knowledge on the different outcome depending of the precise DVT level and extension it is recommended to visualize the DVT in the entire length. In this way, it is more relevant for the clinician to predict a prognostic estimate for the patient with DVT and inform about the risk for future PTS development.

DUS might be difficult and insufficient in obese patient especially in the abdominal region. MRV and CTV are therefore relevant substitutes in the diagnostic armamentarium, also in situations with suspicion of tumor-like processes.

\section{Methods for thrombus removal}

Thrombus removal has been known for many years, initially as a surgical procedure known 50-60 years back first described in Germany. Many years should go before minimal invasive procedures were introduced. In 1991 the first case of catheter-directed thrombolysis (CDT) was published from US (12). A rapid development in the last 20 years has followed with instrumentation and radiographically improved equipment to refine the procedures. Adjunctive devices hoping to speed up the treatment time named pharmacomechanical thrombolysis (PMT or PCDT) and new dedicated venous stents have been introduced.

\section{Lysis and contraindications}

Thrombolysis is achieved by infusion containing components of a plasminogen activating agent to produce plasmin in combination with heparin (unfractionated heparin or LMWH weight-adjusted in therapeutic levels) in a volume of saline ( $\mathbf{F i g . 1}$ ). Plasminogen activators to be used are either urokinase (median 110.000 units/ hour) or ( $r$ )tPA (median $0.6 \mathrm{mg} /$ hour), acting equal sufficiently according thrombus resolution and bleeding rates but with a tendency of shorter treatment time with tPA (13). Today, rtPA is the only agent available in many countries and given in doses from $0.5 \mathrm{mg} /$ hour to $1.2 \mathrm{mg} /$ hour, and the infusion volume varies from $20 \mathrm{ml}$ to $120 \mathrm{ml}(4,14)$. The infusion can be continuous or intermittent as pulse-spray via a catheter with multiple side-holes and tip occlusion. The latter is advantageously combined with high infusion volume to "imitate" a kind of mechanical effect on the thrombus material, also shown from the Copenhagen experience, to be more sufficient than continuous infusion (14).

Coming to this stage of different guidewires and sheaths to penetrate the thrombosed vein segments, lies beyond this chapter. The most important exclusion criteria to thrombolysis are listed in Table 2 ( 1 ).

Some protocols have contained a daily maximum as well of total dosage maximum of tPA or urokinase to reduce bleeding complications. However, this principle might counteract the criteria for a satisfactory rate of thrombus removal before stopping the infusion. More than $50 \%$ thrombus removal has been mostly accepted, whereas $90 \%$ has been the threshold in the Copenhagen experience without maximum of rtPA and also used as threshold in the ATTRACT trial $(4,14,15)$. In this context three important papers including 246 cases have to be mentioned stating that residual post procedural thrombus and lack of patency at 6 months is mostly predictive for worse PTS outcome $(16,17,18)$.

\section{Stenting}

The indication for stenting of persistent iliac obstruction after fulfilled lysis varies as well. Mostly used threshold for stenting is $>50 \%$ remaining obstruction in the CaVenT trial and the ATTRACT trial and in the Copenhagen experience only $10-15 \%$ remaining obstruction was accepted without stenting $(14,15,19)$. Ballooning alone is insufficient because the vein will recoil. The Wallstent, originally constructed for arterial disease, is the most used stent until 
recently, whereas publications now include the new venous designed stents intended to be more flexible with high radial force. We have no evidence to tell us about advantages. Immediate intraprocedural evaluation of lysis before stenting is performed by multiplane venograms. The use of intravascular ultrasound (IVUS) is questioned for this category of patients like stenting for symptomatic patients with non-thrombotic iliac venous lesions (NIVL) or PTS. Only one paper has highlighted the value of IVUS and not venogram for intraprocedural evaluation and to decide continuing thrombolysis (20).

\section{Monitoring}

it is important to monitor the thrombolysis procedure. However, there are no standards for this. Imaging with daily multiplane venograms can definitely qualify the progress of thrombus resolution using the threshold for satisfactory result as mentioned above. The Copenhagen experience has used level of $D$-dimer for guiding as well. The fresher the thrombus is, the faster increases and the higher is the concentration. What matters more is to stop the lytic infusion when normalized concentration is achieved. Sometimes normal venograms have appeared but still with elevated D-dimer, which resulted in further infusion ( $>$ Fig. 1). We have not published specificly on D-dimer measurements as the principle was within our protocol (21). One publication exists trying to correlate D-dimer with outcome (22). D-dimer $>18.4 \mathrm{ug} / \mathrm{ml}$ at 12 hours after onset had a high predictive rate of $>50 \%$ lysis at the end of CDT in 24 patients. However, D-dimer at the end of treatment would have been of greatest interest.

Monitoring for bleeding is also extreme relevant. APTT and fibrinogen are monitored. Careful looking for hematoma, hematuria, vaginal bleeding has to be done regularly as well bleeding from puncture sites. Dedicated crew should be educated for this purpose and also to control that the infusion volume actually comes into the patient, a fault deemed for failure. Threshold for major (stop or reduction of infusion, blood transfusion or intervention) and minor (oozing from puncture site, hematuria etc) has been proposed to be less than $7 \%$ and something higher respectively (1).

\section{Catheter-directed thrombolysis (CDT)}

The first described case with CDT was accompanied with balloon angioplasty. The next important publication from 1994 was with stent implantation for the iliac vein compression syndrome in 14 out of 27 limbs (23). Five years later the first review was given on CDT including 15 studies with 263 patients with iliofemoral DVT (24). The short-term successful outcome varied from $68 \%$ to $100 \%$ patency with clots $>4$ weeks of age to be the most predictive parameter for inferior results. Less than $30 \%$ of patients were stented, and inferior vena cava filters (IVC Filters) were inserted in 49 patients. One death was reported.

The US multicenter registry publication with 303 limbs in 287 patients from 1999 revealed many important informations after 12 months of follow-up (25). Advantage was observed in patients with less than 10 days of DVT history, and patients with a stented iliac vein revealed better patency than without. Poor grade of lysis was predictive for inferior patency. Another lesson was to avoid stenting in the femoral segment.

The first RCT on CDT versus anticoagulation (AC) for iliofemoral DVT was in an Egyptian paper from 2002 with almost 20 patients in each group and 6 months of follow-up (26). Highly significant difference in iliofemoral patency was found in favor of CDT.

Ultrasound enhanced catheter-directed thrombolysis [EKOS] has been introduced to supplement CDT to increase permeability by emitting ultrasound energy along the catheter into the thrombus material in order to achieve higher in-thrombus concentration of the lytic drug. The method has not shown benefit compared to CDT, see below (27).

\section{Pharmacomechanical catheter-directed thrombolysis (PCDT)}

During the following years different adjunctive mechanical procedures were presented, which might shorten the treatment time for lysis in order to minimize the amount of tPA and thereby bleeding. A secondary vision has undoubtedly been a wish for a shorter stay in ICU, while many countries due to medical laws and local hospital directions have decided to have this category of patients under tight observation. The overall background for acting with new devices was mainly the fragmentize thrombus thus making lysis easier. Early results actually did show shorter treatment time, but we are missing long-term results. The most used principle nowadays is with Angiojet (called rheolytic thrombectomy) as a method suitable for lytic infusion and suction/aspiration via the same catheter. The method has shown advantage (PEARL study) in time shortening the procedure with $73 \%$ treated within 24 hours in a 32-center study (US and Europe) with 329 patients and stent rate of $35 \%$ (28).

\section{Thrombus aspiration}

Aspiration (manual) with a syringe as a single principle is attractive. However, often the procedures is performed in combination with lysis afterwards. The most important rapport is from Turkey 2012 (29). A total of 148 patients with acute or subacute iliofemoral DVT revealed patency of $80 \%$ at 3 years follow-up and stent rate of $67 \%$; additional CDT was supplied in $27 \%$. A RCT with 21 patients in each group with iliofemoral DVT treated with thrombus aspiration versus $A C$ showed benefit of aspiration after 1 year according to patency and a modified Villalta score (30).

\section{Surgical thrombectomy}

Surgical approach for iliofemoral thrombosis is an invasive procedure from the groin in general anesthesia. The procedure is well investigated in Hälsingborg more than 20 years ago with the classical RCT of surgery versus anticoagulation including a total of 58 patients with acute iliofemoral DVT. After a substantial loss of patients, a 10-year follow-up consisted of 13 patients in the surgical group and 17 patients in the control group. Patency was better in the surgical group: $83 \%$ versus $41 \%$; $p<0.05$, with tendency of less reflux. It is worthwhile to mention that the thrombectomy 
procedure was without any ballooning or stenting but with a flow stimulating a-v fistula created in the groin (31). A German center with great experience has published important results with surgical thrombectomy in 83 patients with iliofemoral DVT in 2010 (32). In the last patients of the series, the procedure was added with distally infused rtPA via a foot vein. All had an a-v fistula and stent rate was $27 \%$. Life-table analysis showed patency of $75 \%$ after 5 years with moderate PTS rate of $20 \%$.

\section{Recent literature with RCT's and experience from big series since 2010}

The Copenhagen one-center experience with CDT for acute iliofemoral DVT was presented 2011 with 109 patients (31 years, range 15-58) including 111 extremities (33). The lytic infusion mostly given as pulse-spray consisted of $1.2 \mathrm{mg}$ rtPA and weight-adjusted heparin in $120 \mathrm{ml}$ saline per hour via popliteal vein access. Stent rate was $59 \%$. Median follow-up was 71 months. The cumulative rate of patent and reflux-free veins at 6 years was $87.5 \%$. PTS developed in 18 patients (16.5\%) and of those, initial thrombolysis was successful in 13. PTS was associated with worse QOL, although only a few patients developed PTS. Patients with patent veins and sufficient valves have higher QOL scores than patients with reflux and occluded veins.

The Norwegian CaVenT study was the first great constructed randomized controlled trial with 93 patients with proximal DVT treated with CDT versus 108 patients treated with AC involving 4 centers in the region of Oslo with recruitment from 20 hospitals (19). Patients aged 18-75 years with first-time DVT within 3 weeks from symptom onset were enrolled. Previous surgery, trauma $<3$ months and short time immobilization counted for half of risk factors. CDT was used with continuous infusion of rtPA with a maximum of 20 mg per 24 hours for maximum 96 hours. It showed up in the initial venogram that $10 \%$ actually had previous DVT, and half of the patients did not have iliac DVT involvement. AC treatment and compression stockings were recommended for the entire follow-up period. Stent rate was $17 \%$ and even ballooning alone were performed.

At the first evaluation after 24 months, 12 patients were lost to follow-up. The results showed that 37 patients allocated in CDT group presented with PTS $(41.1 \%, 95 \% \mathrm{Cl} 31.5-51.4)$ compared with 55 (55.6\%, 95\% Cl 45.7-65.0) in the control group ( $p=0.047)$. The difference in PTS corresponds to an absolute risk reduction of $14.4 \%$ (95\% Cl 0.2-27.9), and the number needed to treat was 7 (95\% Cl 4-502). Iliofemoral patency after 6 months was reported in 58 patients after CDT $(65.9 \%, 95 \% \mathrm{Cl} 55.5-75.0)$ versus 45 control patients $(47.4 \%, 37.6-57.3, p=0.012)$.

The 5-year results from the CaVenT study were published in 2016 with 87 patients available for CDT and 89 patients available for AC at follow-up (4). Still 37 patients (43\%; $95 \%$ Cl 33-53) allocated to catheter-directed thrombolysis presented post-thrombotic syndrome, compared with 63 (71\%; $95 \%$ Cl 61-79) allocated to the control group ( $\mathrm{p}<0.0001)$, corresponding to an absolute risk reduction of $28 \%$ ( $95 \% \mathrm{Cl} 14-42$ ) and a number needed to treat of 4 (95\% Cl 2-7). Quality-of-life scores did not differ between the treatment groups. The interpretation now concluded that addi- tional catheter-directed thrombolysis resulted in a persistent and increased clinical benefit with CDT.

Results from the large scale and long awaited ATTRACT trial were published in the beginning of 2017 (15). The use of pharmaco-mechanical catheter-directed thrombolysis (PCDT) for proximal deep vein thrombosis was challenged in a randomized study with 336 patients in the thrombus removal group versus 355 in the group treated with best medical therapy (anticoagulation + stockings) alone. Participation included 56 centers in US. The trial did stratify into iliofemoral DVT and femoropoplital DVT but the power calculation was done for all the enrolled patients hypothesizing reduction of PTS from $30 \%$ in the control group to $20 \%$ in the PCDT group. The main outcome in intention-to-treat analysis showed no difference in post-thrombotic syndrome (PTS) after 24 months with Villalta score of $>4: 47 \%$ in the PCDT and $48 \%$ in the control group (risk ratio, 0.96 ; $95 \% \mathrm{Cl} 0.82$ to $1.11 ; \mathrm{p}=0.56$ ). However, moderate-to-severe PTS was more likely in the control group with Villalta score $>9$ : $18 \%$ of patients in the PCDT group versus $24 \%$ of those in the control group (risk ratio, 0.73 ; $95 \% \mathrm{CI} 0.54$ to 0.98 ; $\mathrm{P}=0.04)$. Major bleeding was observed more frequently with PCDT within 10 days $(1.7 \%$ vs. $0.3 \%$ of patients, $p=0.049)$, but no difference in recurrent venous thromboembolism was found over the 24-month follow-up period. The QOL improvement from baseline to 24 months did not differ between the two groups. The analysis of the femoral and iliofemoral cohorts as a single group has been one of the major reasons, that the trial was criticized.

A new publication from the ATTRACT study has now shed more light on the trial with an analysis of the subgroup with iliofemoral disease alone with 196 patients in the PCDT group and 195 patients in the control group (34). These analyses are acknowledged by the authors to be limited by a less substantial power to detect differences in outcome compared to the overall trial and a substantial loss of patients to follow-up.

The outcome still indicates that there is no difference in PTS assessed by Villalta score $>4$ between the thrombus removal group with PCDT and the control group: $49 \%$ versus $51 \%$ respectively (RR 95; $95 \% \mathrm{Cl} 0.78-1.15 ; \mathrm{p}=0.59)$. However, a difference was found in patients with moderate-to-severe PTS in favor of PCDT (Villalta scale $>9$ or ulcer) $18 \%$ versus $28 \%$ (RR 0.65 ; $95 \% \mathrm{Cl} 0.45-0.94$, $\mathrm{p}=0.021$ ) and likewise concerning severe PTS (Villalta scale $>14$ or ulcer) $8.7 \%$ versus $15 \%$ (RR $0.57 ; 95 \% \mathrm{Cl} 0.32-1.01, \mathrm{p}=0.048$ ) as in the main study. Another speculation is highlighted by the stronger significant difference between the groups if VCSS is used as the primary outcome measure compared to Villalta scoring (VCSS $>7$ was $6.6 \%$ versus $14 \%$ in favor of PCDT (RR 0.46 ; $95 \%$ Cl $0.24-0.87$, $p=0.013$ ). From baseline and through 24 months, PCDT led to greater improvement in venous disease specific $Q O L(p=0.029)$, but not in generic $Q O L(p=0.21)$. Finally, and importantly, no difference was found concerning major bleeding and recurrent DVT between the two groups.

A lower rate of PTS was published in two later trials. In one of them, interestingly, CDT was tested in 22 patients versus 23 patients with ultrasound enhanced thrombolysis for acute iliofemoral DVT in 2017, mentioned above (27). PTS scored by Villalta after 1 year was $17 \%$ and $5 \%$ respectively $(p=0.47)$ with stent rate of $80 \%$ in each group. In univariate linear regression analysis, the following baseline characteristics showed a significant association 
> Table 3 Some important studies with alternating length of follow-up after thrombus removal for iliofemoral DVT in the last 10 years, ${ }^{*}=$ moderate-severe PTS, ns= non-significant.

\begin{tabular}{|c|c|c|c|c|}
\hline & Thrombus removal type & Control group/Comparison group & Follow-up & PTS \\
\hline Lindow 2010 (32) & Surgical thrombectomy & none & 60 months & $20 \%$ \\
\hline Broholm 2011 (33) & CDT pulse-spray & none & 72 months & $17 \%$ \\
\hline Haig 2016 (17) & CDT continuous & $A C$ & 60 months & $43 \%$ vs $71 \%$ \\
\hline Engelberger 2017 (27) & CDT US-enhanced & CDT & 12 months & $5 \%$ vs $17 \%$ (ns) \\
\hline Rodriques 2017 (35) & Surgical thrombectomy & CDT & 24 months & $15 \%$ vs $13 \%$ \\
\hline Comerota 2019 (34) & Endovenous removal & $A C$ & 24 months & $18 \%$ vs $28 \% *$ \\
\hline
\end{tabular}

with total Villalta score at 12 months: age $(p=0.021)$, presence of varicose veins $(P<0.001)$ and prior DVT $(P=0.001)$.

The second trial was published in 2018: one group with surgical thrombectomy in 40 patients with acute iliofemoral DVT was compared to CDT (including some patients with PMCDT) in 31 patients aged 18 to 75 years mostly with symptom duration less than 2 weeks (35). No difference was found in PTS, scored with Villalta, $15 \%$ and $13 \%$ patients respectively after 2 years. Stent rate was not given, but stent insertion was more frequent for residual thrombosis in the surgical group. Significantly, more major bleeding was found in the CDT group, which also had a longer hospital stay.

The last paper to present is the non-randomized observational cohort study from Copenhagen (2017) with 203 limbs in 191 patients aged 14-74 years with iliofemoral DVT treated with CDT (14). Median follow-up was 5 years (range: 1 month - 14.3 years]. The stent rate was $52 \%$. Mostly women and left side was affected. The study concentrated on demographics and techniques during treatment being factors with possible influence on outcome. Fifty predefined variables were kept in a database, of which 17 covariates were chosen being clinically and technically most relevant: gender, age, side, stenting, number of stents, caval atresia, caval filter, caval extension of thrombus, thrombus extension below the inguinal ligament, treatment duration, use of pulse-spray or continuous infusion, coagulopathy, child birth after initial thrombosis, use of low molecular weight heparin [LMWH] or heparin, symptoms $<2$ weeks and $>2$ weeks, lifelong anticoagulation, underlying chronic post-thrombotic (subclinical previous DVT) lesions. Six variables were excluded after using non-parametric test and Kaplan-Meier analysis and log rank-test having absence or poor relation with outcome. The remaining 11 variables were included in a multivariate time dependent Cox proportional hazard model. The conclusion was that symptom duration less than 2 weeks, pulsespray infusion technique and no previous DVT did result in better long-term results. The cumulative rate of patients with deep patent veins without reflux at 7 years was $79 \%$. Age, gender, side, IVC atresia, stenting, and lysis duration did not affect outcome. Concerning the stent rate, it had to be interpreted as stent insertions were done sufficiently leaving no significant May-Thurner lesion untreated.

\section{General comments}

It appears clearly, that conflicting results from all these studies do exist. The inclusion criteria vary concerning length of symp- tom duration, previous DVT, amount of lysis and rate of stenting. Furthermore, the methods of thrombus removal are quite different and thresholds for satisfactory lysis and need for stenting are different. A recently published paper, looking at the group with stents, does predict incomplete lysis ( $<50 \%$ ) and stenting below the ligament as strong predictors for PTS (36). This implies the importance of using a sufficient thrombus removal technique during any procedure, which has been highlighted above. It also justifies that the results are given as patency as well with Villalta scoring and QOL assessment.

The use of IVC filters in combination with early thrombus removal has only been touched in this article. Also, the use of this protective device varies a lot. If used temporally the indication mostly has been the visualization of a flagellating thrombus into the IVC itself $(19,37)$. Thrombus removal as such does not stimulate to PE. No peri-procedural PE was found in 69 patients treated with CDT, PCDT or thrombectomy, stent-rate $75 \%$, based on symptoms with following lung perfusion/ventilation scintigraphy or lung spiral CT (38).

Major bleeding was found to be below the threshold mentioned above with a tendency to be more frequent than observed with $A C$ in all the studies. However, in the latest work from the ATTRACT investigators no difference was seen, $1.5 \%$ vs $0.5 \%$ (34). In this context it is also important to inform of no reported death in the last 10 years.

Even in the major recently published ATTRACT study it is impossible to conclude on the best method to achieve patency. This is in strong contrast to the area of varicose vein treatment. No paper in this area does combine different techniques as much as performed in the ATTRACT study. Furthermore, no literature exists to inform of optionally superiority of the new venous designed stents between each other or versus Wallstent.

It has to be mentioned about the positive utility of intermittent pneumatic compression (IPC) during CDT shown in an RCT with less than 15 patients with iliofemoral DVT in each group with and without IPC on the foot and calf during the entire procedure in ward and the operating theatre. Significant less PTS was found in the group with IPC based on greater rate of lysis (39). This principle has been used from the beginning in the Copenhagen experience as one of the very few centers.

Finally, the post-procedural anticoagulation regime has in many of the mentioned trials been recommended for $1-2$ years but with a rather low compliance. However, no consensus exists, but the area has lately been questioned (40). Research has recently shown 
no difference in patency and clinical outcome after 3-12 months vs longer duration with $A C$ in stented patients after CDT, as well as no difference between vitamin $\mathrm{K}$-antagonists and rivaroxaban for the first 3 months period of treatment $(41,42)$.

Many reviews have been published. One of the latest illustrates clearly the problems (43). Even the title is catheter-directed thrombolysis, yet the publication does include the ATTRACT trial with the different techniques included in the study. No doubt that the coming guidelines has to shift from recommendations for each specific thrombus removal technique to a broad term of just: early endovenous thrombus removal. The term: early thrombus removal includes surgical thrombectomy. Some important studies on thrombus removal in the last 10 years are listed in $>$ Table 3.

\section{Conclusion}

The main conclusion is therefore to talk about early thrombus removal as a term instead of any specific modality to be the best option. Obviously, it seems reasonable to offer the most minimal procedure to treat iliofemoral DVT within the first 2 weeks of onset. In a situation where bleeding for sure will be a consequence, then aspiration or even surgical procedure is to prefer. Stenting is mandatory for any persistent iliac obstruction. Removing as much thrombus material as possible and relevant stenting is recommended for optimal prevention of PTS. Use of IVC filters is not advisable, but should be retrievable if inserted in selected cases. The procedures have to be centralized in a close cooperation between interventionalists, vascular surgeons and hematological expertise with knowledge on thrombosis and hemostasis. European guidelines for treatment of venous thrombosis will be published later this year under the auspices of European Society of Vascular Surgery.

\section{Conflict of interest}

No conflicts of interest for this article. The author received fees years ago from Bard, Boston SC, Cook, Servier.

\section{References}

[1] Vedantham S, Sista AK, Klein S] et al. Quality improvement guidelines for the treatment of lower-extremity deep vein thrombosis with use of endovascular thrombus removal. J Vasc Interv Radiol 2014; 25 : 1317-1325

[2] Delis K, Bountouroglou D, Mansfield AO. Venous claudication in iliofemoral thrombosis. Long-term effects on venous hemodynamics, clinical status and quality of life. Ann Surg 2004; 230: 114-126

[3] Kahn SR, Shrier I, Julian JA et al. Determinants and time course of the postthrombotic syndrome after acute deep venous thrombosis. Ann Intern Med 2008; 149: 698-707

[4] Haig Y, Enden T, Grøtte O et al. Post-thrombotic syndrome after catheter-directed thrombolysis for deep vein thrombosis (CaVenT): 5-year follow-up results of an open-label, randomised controlled trial. Lancet Haematol 2016; 3: e64-71

[5] Rabinovich A, Ducruet T, Kahn SR. Development of a clinical prediction model for the postthrombotic syndrome in a prospective cohort of patients with proximal deep vein thrombosis . J Thromb Haemost 2018; 16: 262-270
[6] Caprini JA, Arcelus JI, Hoffman KN et al. Venous duplex imaging follow-up of acute symptomatic deep vein thrombosis of the leg. J Vasc Surg 1995; 21: 472-476

[7] Åkesson H, Brudin L, Dahlström JA et al. Venous function assessed during a 5 year period after ilio-femoral venous thrombosis treated with anticoagulation. Eur J Vasc Surg 1990; 4: 43-48

[8] De Maeseneer MGR, Bochanen N, van Rooijen G et al. Analysis of 1,338 patients with acute lower deep venous thrombosis (DVT) supports the inadequacy of the term "proximal DVT". Eur J Vasc Endovasc Surg 2016; 51: 415-420

[9] ReRoo S, Deatrick KB, Henke PK. The vessel wall: A forgotten player in the post thrombotic syndrome. Thromb Haemost 2010; 104: 681-692

[10] Siudut J, Grela M, Wypasek E et al. Reduced plasma fibrin clot permeability and susceptibility to lysis are associated with increased risk of postthrombotic syndrome. J Thromb Haemost 2016; 14: 784-793

[11] Hach-Wunderle V, Bauersachs R, Gerlach H-E et al. Post-thrombotic syndrome 3 years after deep venous thrombosis in the Thrombosis and Pulmonary Embolism in Out-Patients (TULIPA) PLUS Registry. J Vasc Surg: Venous and Lym Dis 2013; 1: 5-12

[12] Okrent D, Messersmith R, Buckman J. Transcatheter fibrinolytic therapy and angioplasty for left iliofemoral venous thrombosis. J Vasc Interv Radiol 1991; 2: 195-197

[13] Grunwald MR, Hofmann LV. Comparison of urokinase, alteplase and reteplase for catheter-directed thrombolysis of deep venous thrombosis. J Vasc Interv Radiol 2004; 15: 347-352

[14] Foegh P, Jensen LP, Klitfod L et al. Editor's choice: Factors associated with long-term outcome in 191 patients with iliofemoral DVT treated with catheter-directed thrombolysis. Eur J Vasc Endovasc Surg 2017; 53: 419-423

[15] Vedantham S, Goldhaber SZ, Julian JA et al. Pharmacomechanical catheter-directed thrombolysis for deep-vein thrombosis. N Engl J Med 2017; 377: 2240-2252

[16] Comerota AJ, Grewal N, Martinez JT et al. Postthrombotic morbidity correlates with residual thrombus following catheter-directed thrombolysis for iliofemoral deep vein thrombosis. J Vasc Surg 2012; 55: 768-773

[17] Haig Y, Enden T, Slagsvold CE et al. Residual rates of reflux and obstruction and their correlation to post-thrombotic syndrome in a randomized study for deep venous thrombosis. J Vasc Surg Venous Lymphat Dis 2012; 2: 123-130

[18] Avgerinos ED, Hager ES, Naddaf A et al. Outcomes and predictors of failure of thrombolysis for iliofemoral deep venous thrombosis. J Vasc Surg Venous Lymphat Disord 2015; 3: 35-41

[19] Enden T, Haig Y, Kløw NE et al. Long-term outcome after catheterdirected thrombolysis versus standard treatment for acute iliofemoral deep venous thrombosis (the CaVenT study): a randomised controlled trial. Lancet 2012; 379:31-38

[20] Raju S, Martin A, Davis M. Assessment of residual thrombus after venous thrombolytic regiments. J Vasc Surg Venous Lymphat Disord 2014; 2: 148-154

[21] Bækgaard N, Klitfod L, Jørgensen M. Should catheter-directed thrombolysis be monitored? Phlebology 2016; 31 (1 Suppl): 5-10

[22] Luo CM, Wu IH, Chan CY et al. Dimerized plasmin fragment D as a potential biomarker to predict successful catheter-directed thrombolysis therapy in acute deep vein thrombosis. Phlebology 2015; 30: 620-626

[23] Semba CP, Dake MD. lliofemoral deep venous thrombosis: aggressive therapy with catheter-directed thrombolysis. Radiology 1994; 191: 487-494

[24] Grossman C, McPherson S. Safety and efficacy of catheter-directed thrombolysis for iliofemoral venous thrombosis. Roentgenol 1999; 172: $667-672$ 
[25] Mewissen MW, Seabrook GR, Meissner MH et al. Catheter-directed thrombolysis for lower extremity deep venous thrombosis: report of a national multicenter registry. Radiology 1999; 211: 29-49

[26] Elsharawy M, Elzayat E. Early results of thrombolysis vs anticoagulation in iliofemoral venous thrombosis. A randomized clinical trial. Eur J Vasc Endovasc Surg 2012; 24: 209-214

[27] Engelberger RP, Stuck A, Spirk D et al. Ultrasound-assisted versus conventional catheter-directed thrombolysis for acute iliofemoral deep vein thrombosis: 1 year follow-up data of a randomized trial. J Thromb Haemost 2017; 15: 1351-1360

[28] Garcia M], Lookstein R, Malhotra R et al. Endovascular management of deep vein thrombosis with rheolytic thrombectomy: Final report of prospective multicenter PEARL (peripheral use of Angiojet Rheolytic Thrombectomy with a variety of catheter lengths) registry. J Vasc Interv Radiol 2015; 26: 777-785

[29] Oguzkurt I, Ozkan U, Gülcan et al. Endivascular treatment of acute and subacute iliofemoral deep venous thrombosis by using manual aspiration thrombectomy: long-term results of 139 patients in a single center. Diagn Interv Radiol 2012; 18: 410-416

[30] Cakir V, Gulcu A, Akay E et al. Use of percutaneous aspiration thrombectomy vs. anticoagulation therapy to treat acute venous thrombosis: 1-year follow-up results of a randomized clinical trial. Cardiovasc Intervent Radiol 2014; 37: 969-976

[31] Plate G, Eklöf B, Norgren L et al. Venous thrombectomy for iliofemoral vein thrombosis-10-years results of a prospective randomised study. Eur J Vasc Endovasc Surg 1997; 14: 367-374

[32] Lindow C, Mumme A, Asciutto $G$ et al. Long-term results after transfemoral venous thrombectomy for iliofemoral deep venous thrombosis. Eur J Vasc Endovasc Surg 2010; 40: 134-138

[33] Broholm R, Sillesen H, Damsgaard MT et al. Postthrombotic syndrome and quality of life in patients with iliofemoral venous thrombosis treated with catheter-directed thrombolysis. J Vasc Surg 2011; 54 (suppl 6): 18S-25S

[34] Comerota AJ, Kearon C, Gu CS et al. Endovascular thrombus removal for acute iliofemoral deep vein thrombosis. Circulation 2019; 139: $1162-1173$
[35] Rodriguez LE, Aboukheir-Aboukheir A, Figueroa-Vicente R et al. Hybrid operative thrombectomy is noninferior to percutaneous techniques for treatment of acute iliofemoral deep venous thrombosis. J Vasc Surg Venous Lymphat Disord 2017; 5: 177-184

[36] Avgerinos ED, Saadeddin Z, Abou Ali AN et al. Outcomes and predictors of failure of iliac stenting after catheter-directed thrombolysis for acute iliofemoral thrombosis. J Vasc Surg Venous Lymphat Disord 2019; 7: 153-161

[37] Bækgaard N, Broholm R, Just S et al. Long-term results using catheter-directed thrombolysis in 103 lower limbs with acute iliofemoral venous thrombosis. Eur J Vasc Endovasc Surg 2010; 39: 112-117

[38] Protack CD, Bakken AM, Patel $\mathrm{N}$ et al. Long-term outcomes of catheter-directed thrombolysis for lower extremity deep venous thrombosis without prophylactic inferior vena cava filter placement. J Vasc Surg 2007; 45: 992-997

[39] Ogawa T, Hoshino S, Midorikowa $\mathrm{H}$ et al. Intermittent pneumatic compression of the foot and calf improves the outcome of catheter-directed thrombolysis using low-dose urokinase in patients with acute proximal venous thrombosis of the leg. J Vasc Surg 2005; 42: 940-944

[40] Sista AK. Postprocedural management of patients undergoing endovascular therapy for acute and chronic lower-extremity deep venous disease. Tech Vasc Interv Radiol 2014; 24: 1134-1143

[41] Sebastian T, Engelberger RP, Spirk D et al. Cessation of anticoagulation therapy following endovascular thrombus removal and stent placement for acute iliofemoral deep vein thrombosis. VASA 2019; 22: 1-9

[42] Sebastian T, Hakki LO, Spirk D et al. Rivaroxaban or vitamin-K antagonists following endovascular thrombus removal and stent placement for acute iliofemoral deep vein thrombosis. Thromb Res 2018; 172: 86-93

[43] Lu Y, Chen L, Chen J et al. Catheter-directed thrombolysis versus standard anticoagulation for acute lower extremity deep vein thrombosis: A meta-analysis of clinical trials. Clin Appl Thromb Hemost 2018; 24 : 1134-1143 